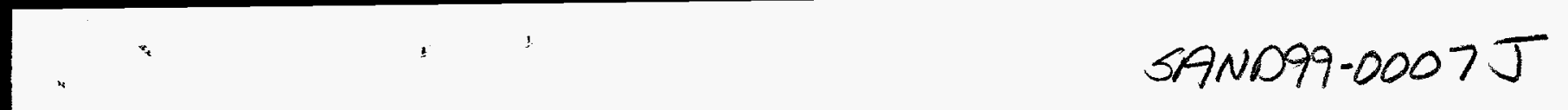

\title{
Magnetic-Field-Induced V-Shaped Quantized Conductance Staircase in a Double-Layer Quantum Point Contact
}

\author{
S. K. Lyo \\ Sandia National Laboratories, Albuquerque, N. M. 87185
}

We show that the low-temperature conductance $(G)$ of a quantum point contact consisting of ballistic tunnel-coupled double-layer quantum well wires is modulated by an in-layer magnetic field $B_{\|}$perpendicular to the wires due to the anticrossing. In a system with a small $g$ factor, $B_{\|}$creates a V-shaped quantum staircase for $G$, causing it to decrease in steps of $2 e^{2} / h$ to a minimum and then increase to a maximum value, where $G$ may saturate or decrease again at higher $B_{\|}$'s. The effect of $B_{\|}$-induced mass enhancement and spin splitting is studied. The relevance of the results to recent data is discussed.

PACS: 72.20.My, 73.50.Jt, 73.61.Ey, 73.40.Kp 


\section{DISCLAIMER}

This report was prepared as an account of work sponsored by an agency of the United States Government. Neither the United States Government nor any agency thereof, nor any of their employees, make any warranty, express or implied, or assumes any legal liability or responsibility for the accuracy, completeness, or usefulness of any information, apparatus, product, or process disclosed, or represents that its use would not infringe privately owned rights. Reference herein to any specific commercial product, process, or service by trade name, trademark, manufacturer, or otherwise does not necessarily constitute or imply its endorsement, recommendation, or favoring by the United States Government or any agency thereof. The views and opinions of authors expressed herein do not necessarily state or reflect those of the United States Government or any agency thereof. 


\section{DISCLAIMER}

Portions of this document may be illegible in electronic image products. Images are produced from the best available original document. 


\section{INTRODUCTION}

It is well known that the low-temperature $(T)$ conductance $(G)$ through a narrow constricted channel known as a quantum point contact (QPC) is quantized in units of $2 e^{2} / h$. [1] This quantization follows from the fact that, in one dimension, each pair of Fermi points on an energy-dispersion curve of a sublevel contributes $e^{2} / h$ per spin to the low-TG, independent of the form of the dispersion. The low- $T G$ is determined by the number of pairs of Fermi points $\nu_{\mathrm{F} \sigma}$ for each spin $\sigma:[1]$

$$
G=\frac{e^{2}}{h} \sum_{\sigma} v_{F \sigma}
$$

We consider a QPC consisting of tunnel-coupled double quantum wells (DQW's) separated by a thin center barrier in the z-direction shown in Fig. 1. The confinement in this direction yields sublevels which will be referred to here as $Q W$ sublevels. The $Q W$ widths $w_{1}, w_{2}$ and the center-to-center distance $d$ are small (e.g., $d \leqslant 300 \AA$ ) allowing only the tunnel-split ground doublets to be occupied at low $T$ 's: higher-energy QW sublevels play no role in this paper except that they can enhance the mass of the electrons in the ground doublets through sublevel mixing at high fields $B_{\|}$as will be shown later. The channel is a few tenths of a micron wide, yielding densely spaced sublevels arising from the channel confinement, namely the confinement in the x-direction. These dense sublevels are defined as channel sublevels. The current flows ballistically along the channel in the y-direction. The purpose of this paper is to show that $B_{\|}$(applied in the x-direction as shown in Fig. 1) creates a V-shaped staircase of the quantized $G\left(B_{\|}\right)$by causing $G$ to decrease initially to a minimum value in steps of $2 e^{2} / h$ in a system with a small $g$ factor and then to increase to a maximum value. For narrow (wide) QW's, $G$ saturates (decreases again) at higher $B_{\|}$'s and 
the saturation (maximum) $G$ is larger (smaller) than $G\left(B_{\|}=0\right)$. A single-QW QPC with $B_{\|}$ in the $\mathrm{z}$ direction has been studied earlier. In this case, $G$ decreases monotonically with increasing $B_{\|}$in steps of $2 e^{2} / h$. $[1,2]$

\section{FIELD-INDUCED ANTICROSSING AND CONDUCTANCE MINIMUM}

The wave function for the structure shown in Fig. 1 is given by $\Psi=e^{i k_{y} y} \phi_{n}(x) \psi\left(z, k_{\mathrm{y}}\right)$, where $n$ is the quantum number for the channel sublevels. The wave function $\phi_{n}(x)$ is determined by the shape of the channel confinement potential and is treated here phenomenologically. The DQW eigenfunction for the z-confinement $\psi\left(z, k_{y}\right)$ is determined by

$$
H=-\frac{\hbar^{2}}{2 m^{*}} \frac{\partial^{2}}{\partial z^{2}}+\frac{\hbar^{2}}{2 m^{*}}\left(k_{y}-\frac{z}{\ell^{2}}\right)^{2}+V(z)+\varepsilon_{n}+\sigma g \mu_{B} B_{\| 1},
$$

where $\varepsilon_{n}$ is the channel-sublevel energy, $\ell=\left(\hbar c / e B_{\|}\right)^{1 / 2}$ is the magnetic length and $V(z)$ is the double-well potential illustrated in Fig. 1. The last term represents the Zeeman energy where $\mu_{\mathrm{B}}$ is the Bohr magneton, $g$ is the $g$ factor, and $\sigma=0,1$. The effect of the Zeeman splitting is negligibly small for GaAs QW's with a small $g=0.44$. The Hamiltonian in Eq. (2) with $\varepsilon_{n}=0$ has been studied earlier [3] and explains many interesting phenomena in two-dimensional DQW's including the $B_{\|}$-dependent magnetoresistance $[3,4-6]$, the anomalous cyclotron mass [7 - 9] and the conductance enhancement in DQW wires with a short mean- free-path [10].

We solve Eq. (2) numerically by transforming it into a 3-point difference equation for several low-lying energy-dispersion curves. Two symmetric (sy1, sy2) and two asymmetric (asy1, asy2) $\mathrm{GaAs} / \mathrm{Al}_{0.3} \mathrm{Ga}_{0.7} \mathrm{As} \mathrm{DQW}$ structures, listed in Table 1, are studied. Figure 2(a) shows the $B_{\|}=0$ eigenvalues of Eq. (2) for the symmetric and antisymmetric ground doublets of syl including five low-lying channel sublevels evenly spaced at energy inter- 
vals of $\delta=0.02 \mathrm{meV}$. [3] The effective mass is given by $m^{*}=0.067$ in the QWs and $m^{*}=$ 0.091 in the barriers in units of the free electron mass. The vertical dots signify an infinite stack of the channel sublevels.

For $B_{\|}>0$ and at the centers $z=\mp d / 2$ of the left and right QWs, the effective wave numbers $\tilde{k}_{\mathrm{y}}=k_{\mathrm{y}}-z / \ell^{2}=k_{\mathrm{y}} \pm d / 2 \ell^{2}$ in Eq. (2) are shifted relative to each other by an amount $\Delta k_{\mathrm{y}}=d / \ell^{2}$. As a result, the energy parabolas of the two QW's are shifted by $\Delta k_{\mathrm{y}}$. The parabolas then anticross due to tunneling, thereby introducing a gap which separates the lower branch from the upper branch as shown in Fig. 2(b) at $B_{\|}=2.7 \mathrm{~T}$. A hump is formed at the lower edge of the gap with a negative curvature at $k_{\mathrm{y}}=0$ in the lower branch at a sufficiently high $B_{\| \text {. }}$. In Fig. 2(c), the energy gap occurs far above the chemical potential $(\mu)$ shown by thick horizontal bars for the density $2.0 \times 10^{7} \mathrm{~cm}^{-1}$. Note that $B_{\|}$deforms the dispersion curve from Fig. 2(a) to Fig. 2(b) by flattening and stretching out the bottom of the lower branch in the $k_{\mathrm{y}}$ direction and introducing additional minima, where the density of states (DOS) diverges as $\varepsilon^{-1 / 2}$. This process transfers states from the upper branch to the region below the gap, thereby lowering $\mu$ closer to bottom of the lower branch. This important point is clearly seen in Figs. 2(a) and 2(b) where $\mu=1.64$ and $1.05 \mathrm{meV}$, respectively, at $B_{\|}=0 \mathrm{~T}$ and $B_{\|}=2.7 \mathrm{~T}$. Here, $\mu$ is relative to the bottom of the lower branch. The number of the occupied states is given by the sum of the lengths of the segments of the channel sublevels below the Fermi level. As a result, it takes a significantly fewer number of channel sublevels to accommodate the electrons, reducing the number of Fermi points and thus $G$.

With further increasing $B_{\|}$, the lower gap edge of the channel sublevels rises, crossing $\mu$, and hence doubling the number of Fermi points from 2 to 4 for each crossed channel sub- 
level and thereby increasing $G$ steadily to a maximum. At high $B_{\|}$'s, $G$ saturates as the two parabolas become separated as shown in Fig. 2(c). For DQW's with very wide wells, however, $G$ decreases again at higher $B_{\|\|}$'s due to the mass enhancement caused by $B_{\|}$-induced intersublevel mixing as will be shown later.

\section{V-SHAPED CONDUCTANCE STAIRCASE}

The $B_{\|}$-dependent $G\left(B_{\|}\right)$obtained from Eqs. (1) and (2) is displayed in Fig. 3 for sy 1 for several electron densities $N \equiv n \times 10^{6} \mathrm{~cm}^{-1}$ and $\delta=0.02 \mathrm{meV}$ and $\delta=0.05 \mathrm{meV}$. For the same $\delta$, the reduction $\Delta G=G\left(B_{\|}=0\right)-G\left(B_{\|}=B_{\min }\right)$ at the $G$ minimum at $B_{\|}=B_{\min }$ is larger for a larger $n$. This is readily understood from the fact that, for a larger $N$, $\mu$ is larger, populating a larger number of channel sublevels, yielding a larger initial $G\left(B_{\|}=0\right)$. Also, it takes a larger $B_{||}$to pass the lower gap edge through $\mu$, yielding a larger $B_{\min }$. In this process, a larger number of the channel sublevels are emptied, resulting in a larger $\Delta G$ at $B_{\|}=B_{\min }$. For the two curves with $\delta=0.05 \mathrm{meV}$ and $\delta=0.02 \mathrm{meV}$ with the same $\pi=$ $10, B_{\min }$ is larger for a larger $\delta$ because $\mu$ is larger for a larger $\delta$. The curves in dotted and thin solid curves show the effect of spin splitting for $g=0.44$ (GaAs) and for $g=2$. $G$ is an odd-integer multiple of $e^{2} / h$ at some $B_{\|}$'s.

Fig. 4 compares $G$ for $\delta=0.02 \mathrm{meV}$ for sy2, asy 1 and asy2. The energy gap $\Delta_{\mathrm{o}}$ of sy 2 at $B_{\|}=0$ is smaller than that of sy 1, yielding a smaller $\mu=0.92 \mathrm{meV}$ compared to $\mu=1.07$ meV of syl for $n=10$. In syl, the occupied channel sublevels consist of only the lower QW doublet because $\Delta_{\mathrm{o}}$ is larger than $\mu$. The levels just under the Fermi level are a rich source of fermi points without costing many occupied states. In sy2 with $\Delta_{\mathrm{o}}$ smaller than $\mu$, however, there are two stacks of occupied channel sublevels, each consisting of the lower and the upper branch, thereby providing two rich sources of Fermi points and yield- 
ing a larger $G(0)$ than that of syl. For sy2, both $B_{\min }$ and the relative drop of $G$ at $B_{\min }$ are smaller than those of sy 1 because the lower gap edge passes through $\mu$ at a lower $B_{\| \text {- }}$

For the asymmetric structure asy $1, \Delta_{\mathrm{o}}=1.71 \mathrm{meV}$ includes the $1.0 \mathrm{meV}$ energy mismatch and is larger than $\Delta_{\mathrm{o}}=1.39 \mathrm{meV}$ of syl. The $G\left(B_{\min }\right)$ is shallow and occurs at a $B_{\min }$ which is somewhat higher than that of sy1. Note that, in asymmetric DQW's, the two noninteracting parabolas intersect twice as they are displaced by $B_{\|}$: the first time, with the same sign of the slopes and, the second time, with opposite signs. The hump develops at the lower gap edge at the higher $B_{\|}$(e.g., $B_{\|}=2.9 \mathrm{~T}$ ) when the two parabolas intersect with opposite signs of the slopes, yielding a higher $B_{\min }$. [3] The effect of spin splitting is also shown in Fig. 4 in dotted and dashed curves. Recently, the $G$-minimum behavior shown in Fig. 3 and 4 with a similar order of magnitude has been observed at $T=0.3 \mathrm{~K}$ in DQW QPC's. [11] However, the quantum steps were not resolved in the preliminary data. The quantum steps smear out at $k_{\mathrm{B}} T \gtrsim \delta$.

The ratios $R \equiv G(\infty) / G(0)$ saturate at large $B_{\|} \equiv B_{\infty}$ for most of the curves in Fig. 3 and 4. The asymptotic behavior of $G\left(B_{\infty}\right) \equiv G_{\infty}$ is reached when the two parabolas in Fig. 2(c) are completely separated and $\mu$ is far below the gap. In DQW's with deep narrow QW's (e.g., sy1, sy2, and asy1) with negligible intersublevel mixing, a larger $B_{\|}$merely displaces the parabolas further away without changing their shapes, $\mu$, or $G$. While most of the $R$ 's in Fig. 3 and 4 are larger than unity, $R$ is less than unity for the bottom curve for asy 1 with $g=2$ and the asy 2 curve in Fig. 4. For the latter, $R$ decreases after reaching a maximum. These anomalies are due to the combination of large Zeeman splitting, field-induced mass enhancement, and the energy asymmetry $\Delta E$ as will be shown below.

The number of the occupied states is calculated by integrating the $\varepsilon^{-1 / 2}$ - type one 
dimensional DOS and is the same at $B_{\|}=0 \mathrm{~T}$ and $B_{\|}=B_{\infty}$ :

$$
2 \sqrt{1-\gamma} \sum_{n=0}^{\infty} \sum_{\sigma^{\prime}=0,1} \sqrt{\mu_{o}-\sigma^{\prime} \Delta_{o}-\varepsilon_{n}}=\sum_{n=0}^{\infty} \sum_{\sigma, \sigma^{\prime}=0,1} \sqrt{\mu_{\infty}-\left(\sigma^{\prime} \Delta E+\varepsilon_{n}+\sigma g \mu_{B} B_{\infty}\right)}
$$

where the square roots vanish for negative arguments and $\mu_{\mathrm{o}}, \mu_{\mathrm{o}}$ are $\mu$ 's at $B_{\|}=0$ and $B_{\|}$ $\equiv B_{\infty} . \Delta E$ is the energy mismatch between the two ground $\mathrm{QW}$ sublevels without tunneling and $1-\gamma$ is the ratio of the mass along the channel at $B_{\|}=0$ and $B_{\| 1} \equiv B_{\infty}$ in the QW's.

For a linear $(\alpha=1)$ and quadratic ( $\alpha=2)$ distributions of the channel sublevel energy $\varepsilon_{n}$ $=n^{\alpha} \delta$ with $\delta \ll \mu_{0}, \mu_{\infty}$, the $\mathrm{n}$-summation can be performed in Eq. (3), yielding

$$
2 \sqrt{1-\gamma} \sum_{\sigma^{\prime}=0,1}\left(x-\sigma^{\prime}\right)^{2-0.5 \alpha} \theta\left(x-\sigma^{\prime}\right)=\sum_{\sigma^{\prime}=0,1} \sum_{\sigma=0,1}\left(y-\sigma \eta-\sigma^{\prime} Z\right)^{2-0.5 \alpha} \theta\left(y-\sigma \eta-\sigma^{\prime} Z\right)
$$

where $x=\mu_{\mathrm{o}} / \Delta_{\mathrm{o}}, \mathrm{y}=\mu_{\infty} / \Delta_{\mathrm{o}}, \eta=\Delta E / \Delta_{\mathrm{o}}, \mathrm{Z}=g \mu_{\mathrm{B}} B_{\infty} / \Delta_{\mathrm{o}}$, and $\theta(x)$ is a unit step function. The ratio $R$ then equals

$$
G(\infty) / G(0)=\frac{\sum_{\sigma^{\prime}=0,1} \sum_{\sigma=0,1}\left(y-\sigma \eta-\sigma^{\prime} Z\right)^{1.5-0.5 \alpha} \theta\left(y-\sigma \eta-\sigma^{\prime} Z\right)}{2 \sum_{\sigma^{\prime}=0,1}\left(x-\sigma^{\prime}\right)^{1.5-0.5 \alpha} \theta\left(x-\sigma^{\prime}\right)} .
$$

The ratio $R$ is plotted as a function of $x=\mu_{\mathrm{o}} / \Delta_{\mathrm{o}}$ in Fig. 5 for $\varepsilon_{n}=n \delta$. We first discuss $R$ for $Z=0$. In this case, $R \geqq 1$ for $\gamma=0$ as seen from Fig. 5(a) and is consistent with the results in Fig. 3. For symmetric DQW's (i.e., $\eta=0$ ), $R$ equals $R=2^{1 / 3}=1.26$ and $R=\sqrt{2}=$ 1.41, respectively, for $\alpha=1$ and $\alpha=2$ for $x=\mu_{\mathrm{o}} / \Delta_{\mathrm{o}} \leqq 1$ and decreases monotonically above $x>1$ approaching unity at large $x$. For asymmetric DQW's (i.e., $\eta>0$ ) with $\gamma=0, R$ equals unity for $x \leqq \eta$, increases monotonically to a maximum at $x=1$ and decreases monotonically approaching unity at large $x$.

The quantity $\gamma$ equals $\gamma \approx 2 \hbar \omega_{\mathrm{c}}<1|z| 2>^{2} /\left(E_{12}{ }^{*} \ell^{2}\right)$ for the single-QW sublevels $\|>$ and $12>$ with parity. Here $\hbar \omega_{\mathrm{c}}$ is the cyclotron energy and $E_{12}{ }^{*}=E_{12}+\hbar \omega_{\mathrm{c}}\left(\left\langle 2\left|z^{2}\right| 2\right\rangle-\right.$ 
$\left.<1\left|z^{2}\right| 1>\right) / 2 \ell^{2}$ is the level separation. A similar effect was found earlier using a different approach. [12] $\gamma$ increases the DOS, reducing the number of occupied channel sublevels and thus $R$ as shown by the dashed curves in Fig. 5(a). This effect is important in wide QW's such as asy2 where $\gamma=0.09$ at $10 \mathrm{~T}$ due to $B_{\|}$-induced intersublevel mixing. For the asy 2 curve in Fig. 4 with $x=0.63$ and $\eta=0.68, R$ is smaller than unity as shown in Fig. 5(a): $\gamma$ increases with $B_{\|}$yielding a maximum in $G$.

For $Z>0, R$ is invariant under the interchange $\eta \leftrightarrow Z$. The effect of $Z$ is shown in Fig. 5(b), where $Z=0.11$ and $Z=0.5$ correspond, respectively, to the spin splitting at $B_{\infty}=6 \mathrm{~T}$ for $g=0.44, g=2$ and $\Delta_{0}=1.39 \mathrm{meV}$. An interesting aspect of the result in Fig. 5(b) is that, when $x$ is less than the lesser of $(\eta, Z)$ times $2^{-2 / 3}, R$ equals $R=2^{-1 / 3}$ which is about $20 \%$ less than unity.

The conductance displayed in Figs. 3 and 4 is consistent with the results shown in Fig. 5. The $G$ ratios obtained from the curves with $n=10$ in Figs. 3 and 4 agree with those from Fig. 5(a) at least to three significant figures. For the low density case $n=2.5$ in Fig. 3, however, the ratio $R=1.33$ shows a 5\% deviation from the theoretical value $R=1.26$ in Fig. 5(a) because the continuum approximation is less accurate for small $\mu_{0}=0.42 \mathrm{meV}$. The effect of spin splitting on $G$ in Figs. 3 and 4 is small for GaAs QW's. However, for $g=$ 2, there is a significant effect at high $B_{\|}$'s. An especially interesting aspect of the Zeeman splitting occurs when it is combined with the asymmetry $\eta>0$. In this case, $R$ can become less than one as shown in Fig. 5(b) and occurs for the bottom curve in Fig. 4.

For large $\delta$, fewer channel sublevels are populated, yielding a smaller $G$ as seen in Fig. 3. The $\delta$ dependence of $G$ is displayed for $g=\gamma=0$ in Fig. 6 for sy 1 with $\pi=2.5$ and $\Delta_{0}=$ $1.39 \mathrm{meV}$ for $B_{\|}=0,2.2$, and $6 \mathrm{~T}$. The dashed curves there represent the analytic results for 
$\alpha=1$ obtained from the continuum approximation at $B_{\|}=0$ and $B_{\|} \equiv B_{\infty}$ :

$$
x^{3 / 2}+\left(x-\Delta / \Delta_{o}\right)^{3 / 2} \theta\left(x-\Delta / \Delta_{o}\right)=\delta / \delta^{*}
$$

and

$$
G=\frac{2 e^{2} \Delta_{o}}{h \delta}\left[x+\left(x-\Delta / \Delta_{o}\right) \theta\left(x-\Delta / \Delta_{o}\right)\right]
$$

where $x=\mu / \Delta_{\mathrm{o}}$ and $\delta^{*}=0.688 \Delta_{\mathrm{o}}\left(m^{*} \Delta_{\mathrm{o}}\right)^{1 / 2} / \pi$, Here $\delta$ and $\Delta_{\mathrm{o}}$ are in units of meV and $m^{*}$ is the effective mass along the channel in the QW's and $\Delta=\Delta_{\mathrm{o}}$ at $B_{\|}=0$ and $\Delta=\Delta E$ at $B_{\|}$ $\equiv B_{\infty}$. Note that $G(0)$ is determined solely by $\delta, n, m^{*}$, and $\Delta_{0}$, while $G(\infty)$ requires an additional parameter $\Delta E$. In Fig. $6, G$ drops as $\delta^{-1 / 3}$ for the dashed curves in the regime $\delta<$ $\delta^{*}=0.12 \mathrm{meV}$

\section{SUMMARY}

We have shown that the low- $T G$ of a QPC consisting of coupled DQW wires is modulated by a magnetic field $B_{\|}$perpendicular to the wires due to the anticrossing. $B_{\|}$is shown to create a $V$-shaped quantum $G$ staircase, causing it to decrease to a minimum in steps of $2 e^{2} / h$ and then increase to a maximum value, where $G$ saturates (decreases again) for narrow (wide) QW's at higher $B_{\|}$'s. For the former (latter) case, the saturation (maximum) $G$ is larger (smaller) than $G$ at $B_{\|}=0$. The effect of $B_{\|}$-induced mass enhancement and spin splitting was examined.

\section{Acknowledgment}

The author thanks Dr. J. S. Moon, J. A. Simmons and M. Blount for the discussions about the double quantum wire structures and for making their data available to him prior to publication. Sandia is a multiprogram laboratory operated by Sandia corporation, a Lockheed Martin Company, for the U.S. DOE under Contract No.DE-AC04-94AL85000. 


\section{References}

1. C. V. J. Beenaker and H. van Houton, in Solid State Physics (Vol 44): Semiconductor Heterostructures and Nanostructures, edited by H. Ehrenreich and D. Turnbull (Academic, New York, 1991) and references therein.

2. B. J. van Wees et al., Phys. Rev. B 38, 3625 (1988).

3. S. K. Lyo, Phys. Rev. B 50, 4965 (1994).

4. J. A. Simmons, S. K. Lyo, N. E. Harff and J. F. Klem, Phys. Rev. Lett. 73, 2256 (1994).

5. A. Kurobe al., Phys. Rev. B 50, 4889 (1994).

6. O. E. Raichev and F. T. Vasko, Phys. Rev. B 53, 1522 (1996).

7. J. A. Simmons, N. E. Harff, and J. F. Klem, Phys. Rev. B 51, 11156 (1995).

8. S. K. Lyo, Phys. Rev. B 51, 11160 (1995).

9. I. S. Millard et al., J. Phys.: Condens. Matter 9, 1079 (1997)

10. S. K. Lyo, J. Phys.: Condens. Matter 8, L703, 1996.

11. J. S. Moon et al. (unpublished), S. T. Stoddart et al., Proc. of ICPS-24, in press.

12. L. Smrcka and T. Jungwirth, J. Phys.: Condens. Matter 6, 55 (1994). 
Table 1 DQW's with well widths $w$, center-barrier width $t$, well depths $V_{1}, V_{2}$, and $B_{\|}=0$ energy gap $\Delta_{\mathrm{o}}$.

\begin{tabular}{cccc}
\hline \hline structure & $\begin{array}{c}w / t \\
(\AA)\end{array}$ & $\begin{array}{c}V_{1} / V_{2} \\
(\mathrm{meV})\end{array}$ & $\begin{array}{c}\Delta_{\mathrm{o}} \\
(\mathrm{meV})\end{array}$ \\
\hline sy1 & $150 / 25$ & $280 / 280$ & 1.39 \\
sy2 & $135 / 40$ & $280 / 280$ & 0.55 \\
asy1 & $150 / 25$ & $280 / 279$ & 1.71 \\
asy2 & $200 / 20$ & $280 / 279$ & 1.46 \\
\hline \hline
\end{tabular}


Figure Captions

Fig. $1 \mathrm{~A} \mathrm{DQW}$ (gray sheets) QPC $(\| \mathbf{y})$. The field $B_{\|}(\| \mathbf{x})$ is perpendicular to the channel and the growth (z) direction and $d \ll$ channel width.

Fig. 2 Energy vs. $k_{y}$ for syl for $g=0$ for five low-lying channel sublevels spaced at intervals of $\delta=0.02 \mathrm{meV}$ (not to the scale). The dotted lines signify higher-energy channel sublevels. The horizontal bars denote $\mu$ for $N=2.0 \times 10^{7} \mathrm{~cm}^{-1}$.

Fig. 3 Quantized $G$ vs. $B_{\|}$for syl. Thick solid, dotted, and thin solid curves are for $g=0$, 0.44 (GaAs), and 2, respectively.

Fig. 4 Quantized $G$ vs. $B_{\| \mid}$for structures sy2, asy1 and asy2 (upper scale). Dotted and thin solid curves show the effect of spin splitting.

Fig. 5 The conductance ratio vs. $\mu_{\mathrm{o}} / \Delta_{\mathrm{o}}$ for uniformly spaced channel sublevels in the absence of the (a) Zeeman splitting and (b) mass enhancement.

Fig. 6 Quantized $G$ vs. $\delta$ for sy 1 and $g=\gamma=0$. The dashed curves are the analytic results from Eqs. (6) and (7) at $B_{\|}=0$ and $B_{\|}=B_{\infty}$. 


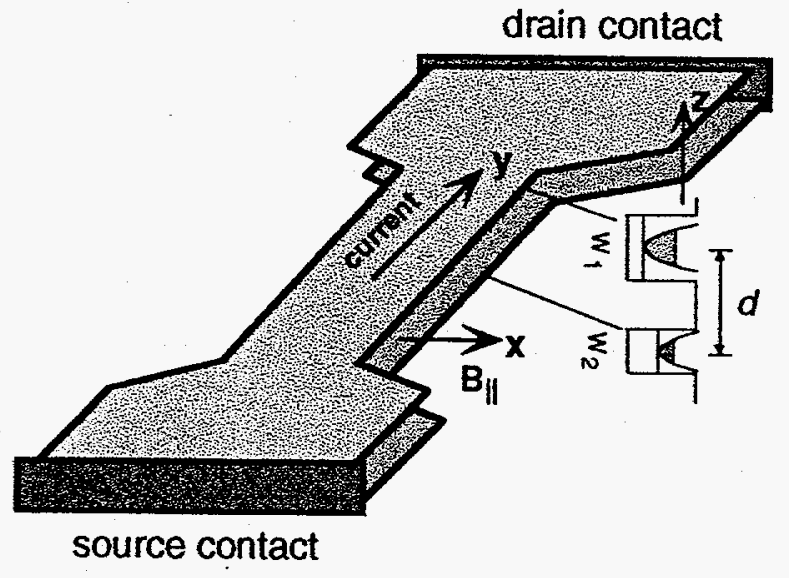

Fig. 1 


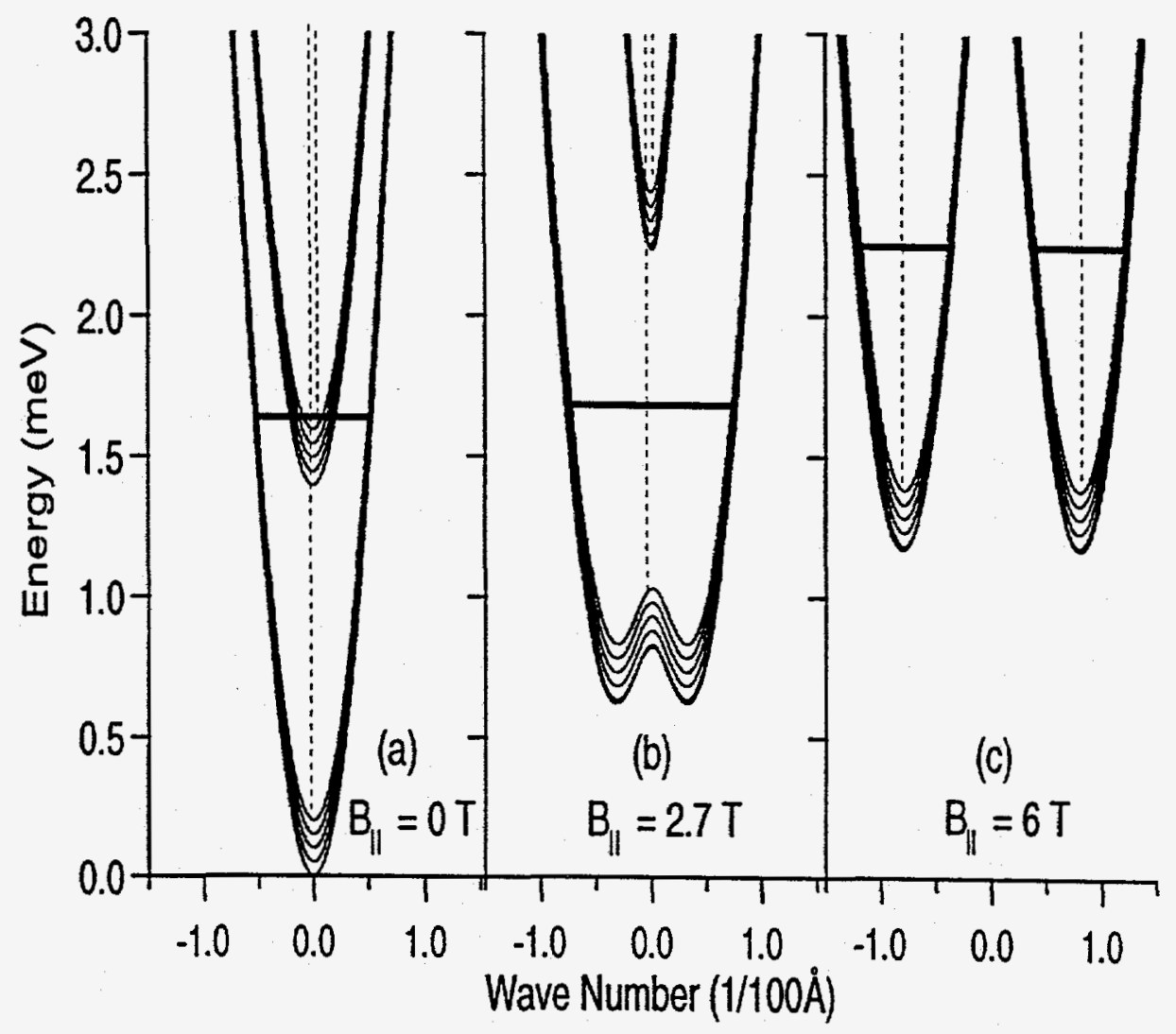

Fig. 2 


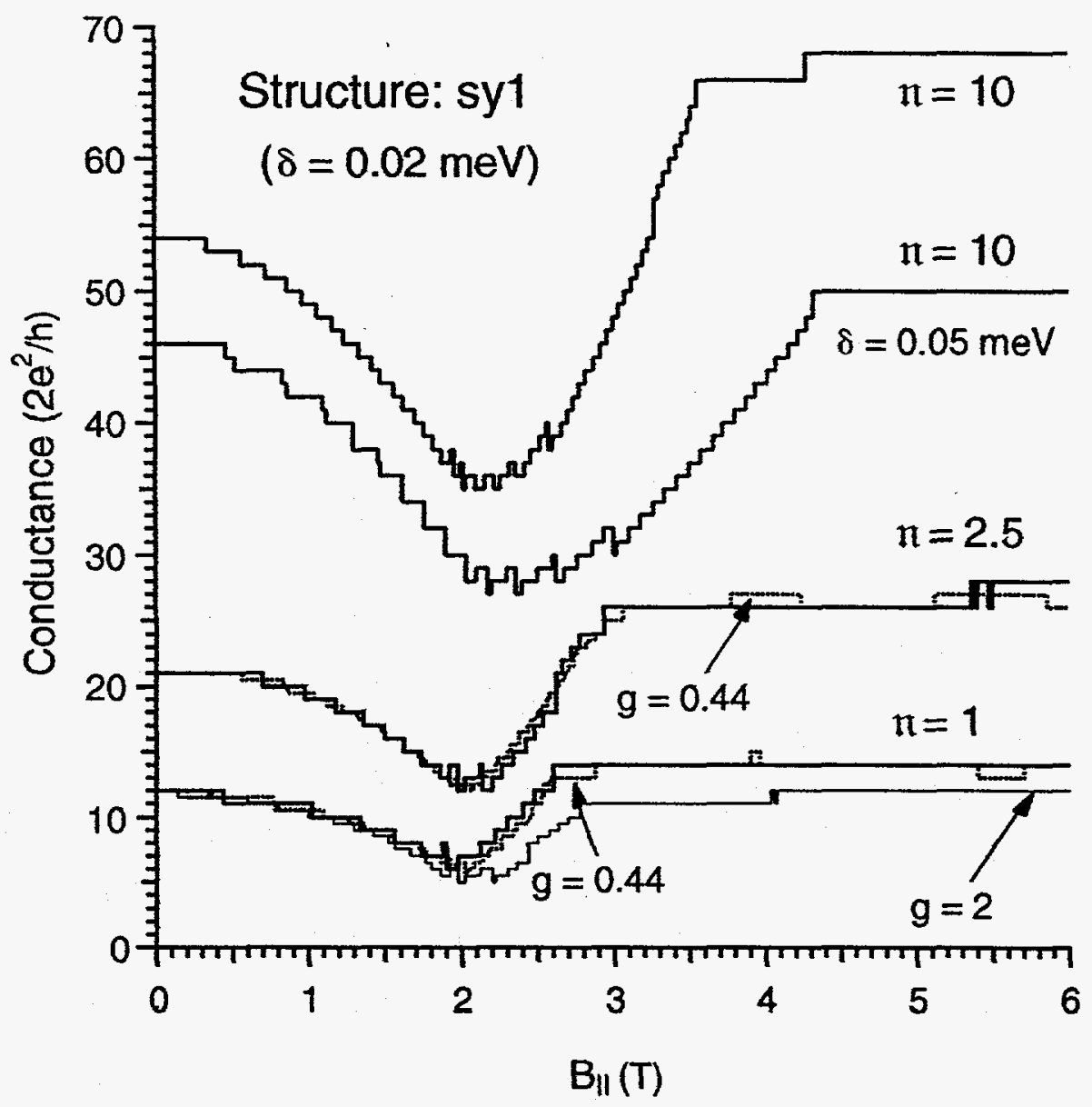

Fig. 3 


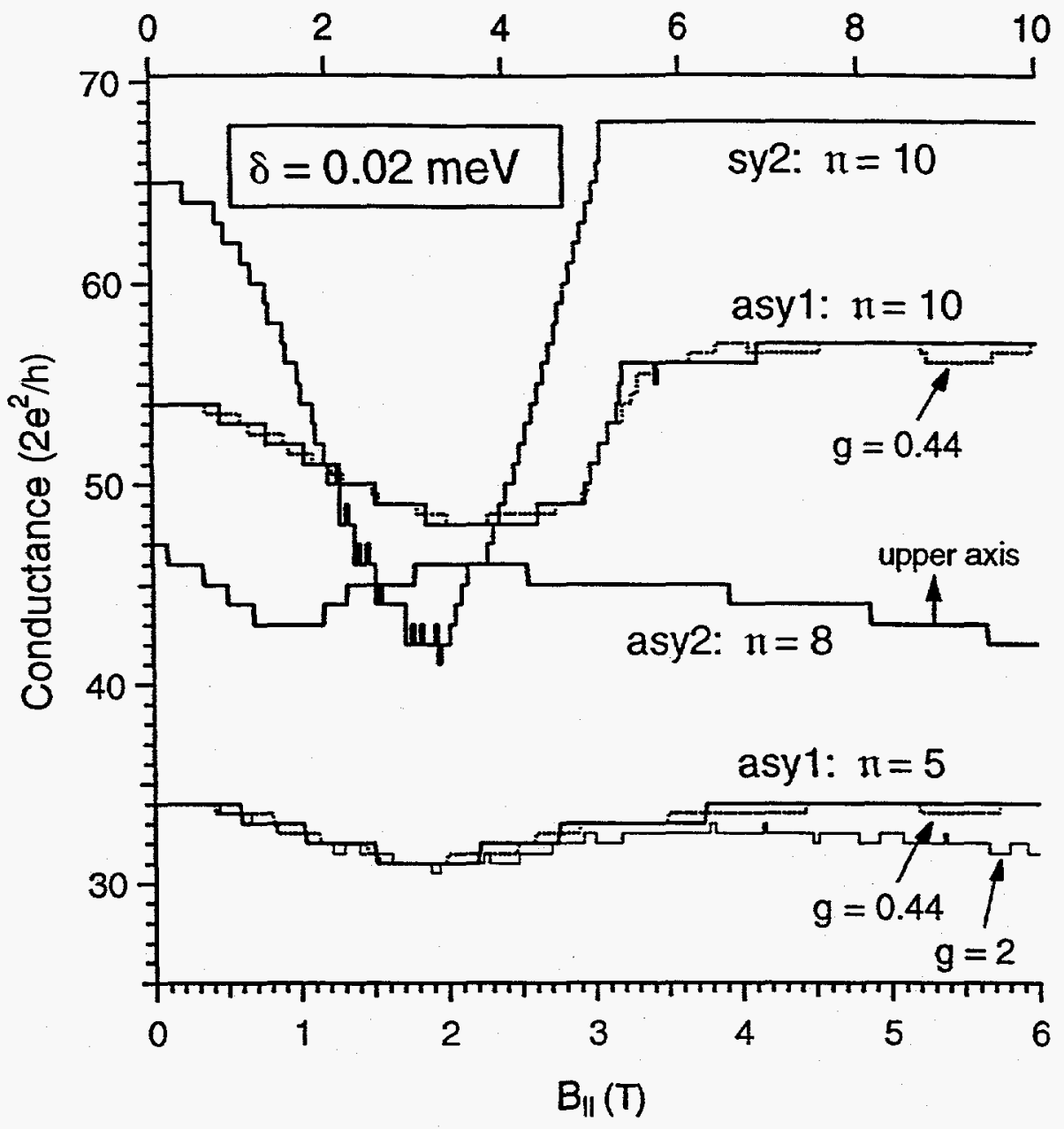

Fig. 4 


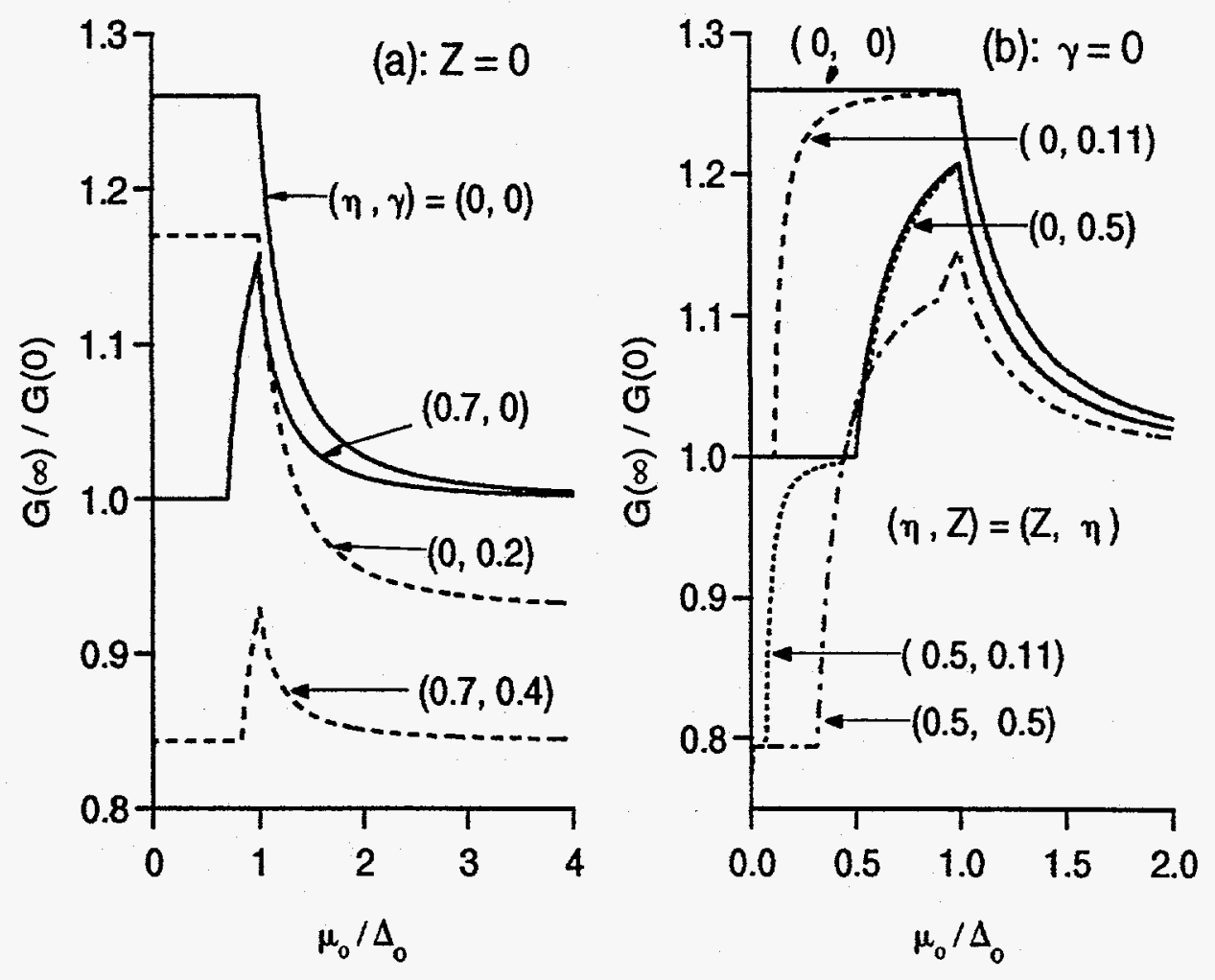

Fig. 5 


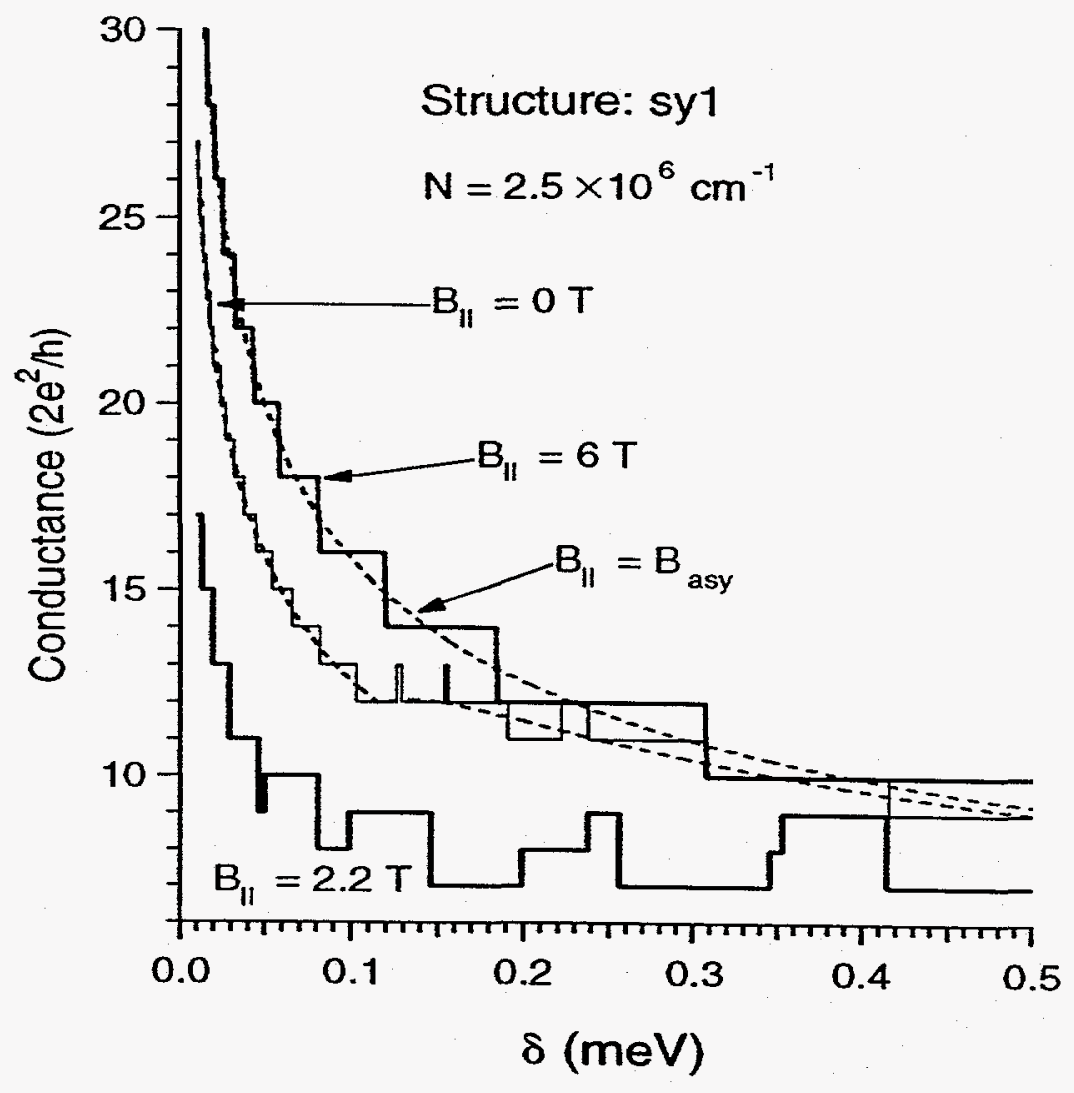

Fig. 6 